\title{
A Haptic Guidance Tool for CT-Directed Percutaneous Interventions
}

\author{
E. Hagmann ${ }^{1}$, P. Rouiller ${ }^{1}$, P. Helmer ${ }^{1}$, S. Grange ${ }^{1}$ and C. Baur ${ }^{1}$ \\ ${ }^{1}$ VRAI Group, Institut de production et robotique, Swiss Federal Institute of Technology, Lausanne, Switzerland
}

\begin{abstract}
We have developed a new navigation approach for computer-assisted interventional radiology. Our system combines a virtual reality display with high-fidelity haptic rendering to provide assistance and guidance of the medical gesture. Specifically, the system is designed to improve the accuracy of blind needle placement within tissues. The proposed technique actively helps the surgeon while keeping him in control of the procedure. We have recently developed an experimental setup for CT-guided biopsy. The setup features a high-precision haptic device connected to the biopsy needle, combined with a graphical interface. The haptic system guides the surgeon's hand to the target tissue based on CT data, whereas a real-time, graphical visualization of the tool trajectory provides navigation information. The setup requires rigid registration of the patient with respect to the haptic interface. Tests have been performed in the presence of radiologists to validate the proposed concept, and early results show that the system is easy to use and requires little training. We are planning to conduct clinical testing in the near future to quantitatively assess system performance.
\end{abstract}

Keywords-CT-directed biopsy, force-feedback, force guidance, haptic guidance

\section{INTRODUCTION}

A common problem in traditional interventional radiology is to accurately access target tissues within the patient's body. In some cases, the presence of organs and bone structures makes it difficult to perform gestures that inherently require significant dexterity. Also, minimizing intervention duration is essential to limiting intervention cost as well as pain and trauma for the patient.

\section{A. Problem approach}

Several approaches exist to augment a surgeon's perception and ability to perform accurate gestures. While these methods share common goals, their implementation is dependant on application constraints and the physicians involved [1, 2]. Three approaches are commonly used:

- Robots [8, 9, 14, 15] automatically perform gestures planned by the user, who does not have direct, real-time physical control over the intervention.

- Navigation tools $[12,13]$ measure tool (and possibly target) positions to provide visual feedback to the user. These tools do not improve the precision nor the stability of the gesture from a mechanical perspective.

- Tele-operated systems $[5,16,17]$ augment the accuracy and stability of the medical gesture. Typically, the user remotely controls a tele-operated tool, such as a joystick, from outside the surgical field. The surgeon is not in direct contact with the intervention scene.

We propose a shared control approach following $[3,7]$ that allows the interventional radiologist to stay in direct contact with the intervention scene and keep full control over the gesture. The tool used for the procedure is mounted on a mechanical arm that is responsible for two tasks: (1) it provides real-time measurement of the tip position with respect to the target and (2) it provides tactile feedback to guide the surgical gesture. To validate this concept, we have developed a setup for CT-based biopsy procedures.

\section{B. Biopsy application}

Conventional biopsy procedures require significant dexterity, as the target identified on a CT-scan is typically less than $10 \mathrm{~mm}$ wide. Accurate trajectory planning based on a set of parallels CT slices is therefore not intuitive. Manual introduction of the biopsy guide usually requires several iterations to control and adjust the tool trajectory during the procedure. At each iteration, a new CT scan must be performed, leading to longer, more costly interventions.

Our system allows for faster, easier execution of biopsy procedures [3]. A graphical user interface displays navigation data and assists trajectory planning via a planar view of the trajectory, whereas high-fidelity haptics make it possible to reach the target in a single iteration.

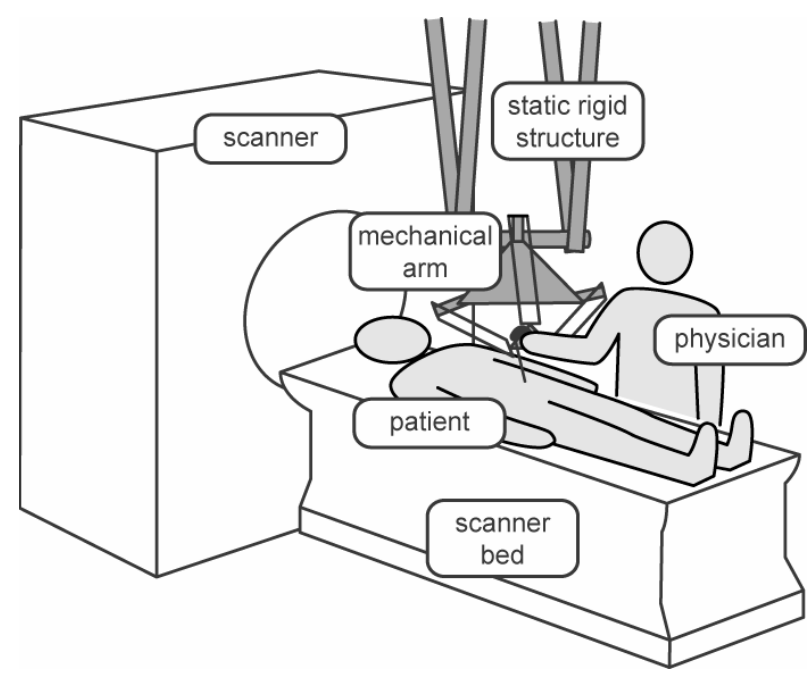

Fig. 1. System configuration concept 


\section{Methodology}

\section{A. Mechanical design}

The system is designed to be used in a radiology theater with a CT scanner and can easily be extend to other imaging systems like C-arm, X-rays and ultrasound. The mechanical structure is mounted on the ceiling to avoid reducing scanner accessibility (Fig. 1). The 5-degree-of-freedom (DOF) device is a combination of a Delta mechanical structure providing translation and guidance forces and a dedicated wrist, which provides orientation and guidance torques.

\section{B. System description}

We have implemented a prototype of the system on top of a commercially available haptic device to validate the concept (Fig. 2).

The Delta Haptic Device (DHD) is a parallel structure that provides 3-DOF in translation. The tool-holder is mounted on a 2-DOF spherical SCARA wrist (Fig. 3), serially attached to the DHD. This configuration makes it possible to rotate the tool around a projected, virtual point and provides a large angular workspace.

The user interface, which we call the BiopsyNavigator [3], shows two different views in real-time: (1) a 3D visualization of the biopsy needle with respect to the patient, and (2) a planar view of the current trajectory, which is aligned along the tool orientation (Fig. 4). The planar view explicitly shows the tissues affected by the chosen trajectory and the distance between the needle and the target. The user can switch between the different modes of the biopsy procedure GUI using buttons mounted on the tool.

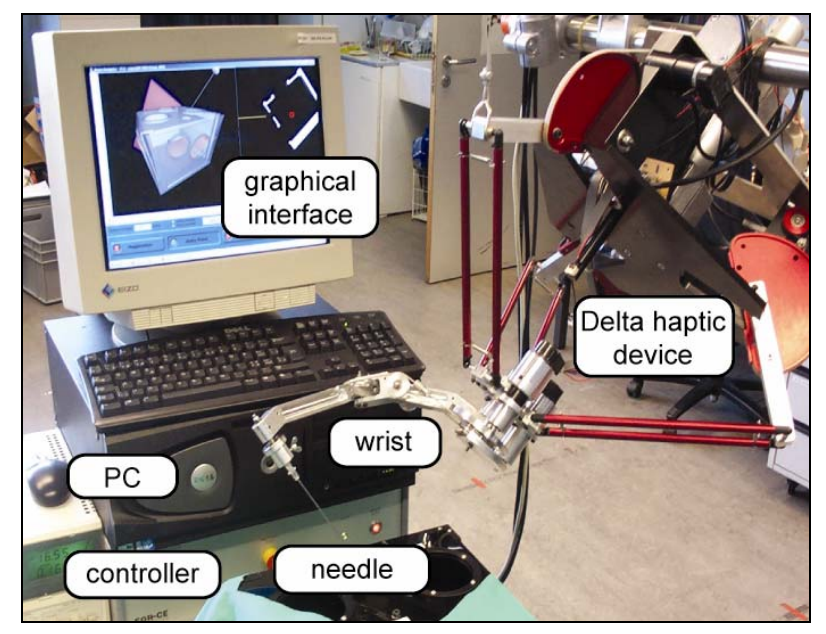

Fig. 2. CT-Guided biopsy protype

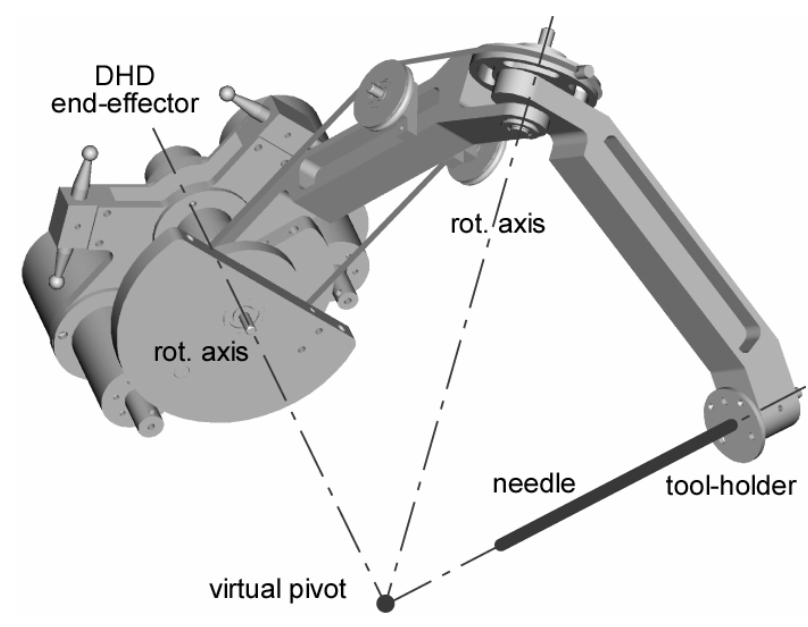

Fig. 3. Dedicated spherical SCARA wrist

\section{Procedure}

The data necessary to the intervention, namely the CT-scans of the zone of interest, are acquired pre-operatively. The computer then builds a 3D model from the scan data, and the radiologist defines the target tissue on the scan images.

The procedure first requires a rigid registration of the 3D model with respect to the patient. To do this, we use Horn's approach [4], which relies on the correspondence of points in the virtual model measured with the haptic setup in the real world.

In neutral state, the system compensates for gravity forces and maintains the tool-holder and the needle balanced. The user can easily move and release the needle without it falling. This improves the stability of the gesture.

In the next phase of the procedure, the haptic system constraints the needle axis to intersect with the target tissue. The radiologist uses the tool-holder to navigate around the target and to chose the preferred introduction trajectory (based on the visualization data from the user interface).

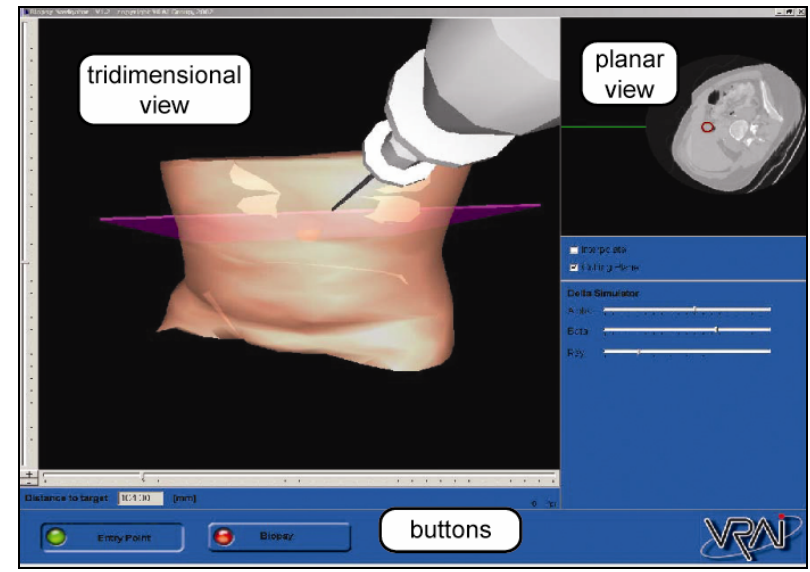

Fig. 4. Prototype graphical user interface 


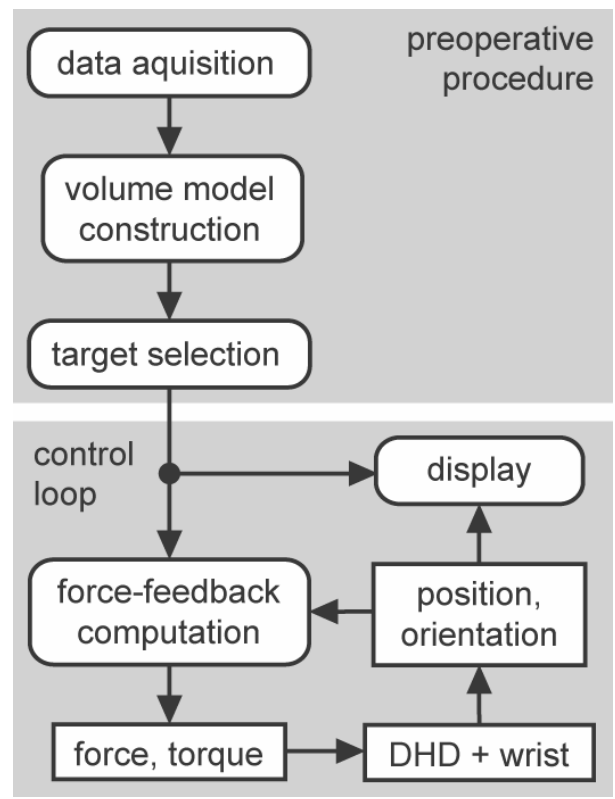

Fig. 5. Schematic of system information flow

Once the optimal trajectory has been defined, the system constraints the needle along the introduction axis. The user can proceed to introducing the needle, using the interface to observe the gesture progress and the distance to the target. At any point in time, the user can modify the trajectory by applying a force greater than the constraint applied by the haptic device.

When the tip of the guide reaches the target, the haptic device actively stops the gesture to prevent overshoot.

\section{RESULtS}

We have characterized the system in terms of positioning accuracy. The DHD is capable of a linear resolution of $0.1 \mathrm{~mm}$, while the wrist achieves an angular resolution of $0.02 \mathrm{deg}$. With no force applied on the toolholder, the absolute precision is $5 \mathrm{~mm}$ in translation and 4 deg in orientation. We also measured the system total compliance. For a pure force of $10 \mathrm{~N}$ applied on the toolholder, accuracy is $10 \mathrm{~mm}$ in translation and $6 \mathrm{deg}$ in orientation.

We asked a physician to qualitatively evaluate the system in an artificial testbed : an empty, opaque box with several openings that contained a physical target for the user to reach. In our tests, we observed the following :

- A $6 \mathrm{~mm}$ target was reached blindly with a mean time of $60 \mathrm{~s}$.

- Shared control significantly improves usability.

- The system use is very intuitive, no particular training is required.
- The mechanical device configuration is not compatible with right- and left-handed use. The mechanical structure can hinder the gesture.

- Mechanical friction is higher in the DHD than in the wrist, this cause tactile inhomogeneity and sensibility perturbation during needle introduction.

\section{DISCUSSION}

System's resolution is sufficient to provide smooth force-feedback. In case of biopsy procedure, the size of a typical CT detected tumor is $5 \mathrm{~mm}$. The measured positioning accuracy of the mechanical structure are sufficient, as the user will not apply an effort opposite to the tactile feedback.

Rigid registration method that we currently use does not take soft tissue movement, such as variations caused by the patient breathing, into account. Thus, the registration is only valid if the CT scans and the needle insertion are performed during similar breathing cycle states. During a typical biopsy procedure, which is performed on empty lungs, rigid registration is sufficiently accurate.

The actual system calibration is performed experimentally. For applications that require higher accuracy, we do propose a calibration by parameter identification [11]. Moreover, an improvement of the structure stiffness is possible through mechanical modifications of the DHD and the wrist.

We are currently redesigning the mechanical structure based on observations from the qualitative test. To improve system modularity and compatibility, we intend to use a mobile positioning base instead of the current static structure. To minimize clutter for the user, we propose to increase the length of the DHD's parallel links and reduce the base plate size, as well as improving stiffness. To reduce mechanical friction, the improved version will feature brushless motors. If it is not sufficient, we plan to add a 1DOF sliding tool-holder in order to allow the DHD to remain stationary during needle introduction. 


\section{CONCLUSION}

We have developed a new navigation system for computer-assisted interventional radiology. We have shown that combining a high-fidelity haptic interface with visual navigation information can improve the ease and accuracy of performing certain surgical gestures.

We have implemented a demonstrator for CT-based biopsy procedures. A first prototype was used to conduct qualitative tests with a physician to validate the proposed concept. The same approach could benefit other interventional radiology procedures such as cryo-therapy, thermo-therapy and radiofrequency-therapy as well as surgical procedures (e.g. neurosurgery).

In the future, we intend to test the system on organic tissue and to perform a complete assisted procedure in a surgical setting.

\section{ACKNOWLEDGMENT}

This research was performed in collaboration with the University Hospital of Geneva (HUG) and was supported by a grant from the Swiss NSF Computer Aided and Image Guided Medical Interventions (NCCR CO-ME) program.

\section{REFERENCES}

[1] J. Troccaz, "Quels Robots pour les Application MédicoChirurgicales?”, Repères, vol. 23, RobAut, Faculté de Médecine du CNRS, 1998.

[2] K. Cleary and C. Nguyen, State of Art in Surgical Robotics : "Clinical Applications and Technology Challenges", Georgetown University, Computer Aided Surgery, 2001.

[3] G. Marti, P. Rouiller, S. Grange and C. Baur, "Biopsy Navigator : a smart haptic interface for interventionel radiological gestures", International Congress Series, vol 1256, Proceedings of Computer Assisted Radiology and Surgery, 2003.

[4] B.K.P. Horn, "Closed-form Solution of Absolute Orientation using Unit Quaternions", Journal of the Optical Society of America, vol. 4, pp. 629-642, 1987.

[5] Z. Nawrat, L. Podsedkowski, K. Mianowski, P. Wroblewski, P. Kostka, M. Baczynski, Z. Malota, G. Granosik, E. Jezierski, A. Wroblewska and Z. Religa, "RobIn Heart in 2002 - Actual State of Polish Cardio-Robot", Third international Workshop on Robot Motion and Control, Foundation of Cardiac Surgery Developpement in Zabrze, Technical University of Lodz and Warsaw University, 2002.

[6] G. Megali, O. Tonet, C. Stefanini, M. Boccadoro, V. Papaspyropoulos, L. Angelini and P. Dario, A Computer-Assisted Robotic Ultrasound-Guided Biopsy System for Video-Assisted Surgery, MiTech Lab, Scuola Superiore Sant'Anna, Pisa, Italy, 2002

[7] R. Taylor, P. Jensen, L. Whitcomb, A. Barnes, R. Kumar, D Stoianovici, P. Gupta, Z. X. Wang, E. deJuan and L. Kavoussi, "A Steady-Hand Robotic System for Microsurgical Augmentation", International Journal of Robotics Research, vol. 18, pp. 1201-1210, 1999.

[8] G. Fichtinger, T. L. DeWeese, A. Patriciu, A. Tanacs, D. Mazilu, J. H. Anderson, K. Masamune, R. H. Taylor and D. Stoianovici, "System for Robotically Assisted Prostate Biopsy and Therapy with Intraoperative CT Guidance", Academic Radiology, vol. 9, pp. 60-74, no. 1, 2002.
[9] K. Cleary, D. Stoianovici, N. Glossop, K. Gary, S. Onda, R. Cody, D. Lindisch, A. Stanimir, D. Mazilu, A. Patriciu, V. Watson and E. Levy, CT-Directed Robotic Biopsy Testbed : Motivation and Concept, Georgetown University Medical Center of Washington and Johns Hopkins Medical Institutions of Baltimore, 2001.

[10] J.B. Ra, S.-M. Kwon, J.K. Kim, J. Yi, K.H. Kim, H.W. Park, K.-U. Kyung, D.-S. Kwon, H.S. Kang, S.T. Kwon, L. Jiang, J. Zeng, K. Cleary and S.K.Mun, "Spine Needle Biopsy Simulator Using Visual and Force Feedback", CNU in Daejeon, SNU in Seoul and Georgetown University at Washington, Computer Aided Surgery, 2002.

[11] J.M. Renders, E. Rossignol, M. Becquet, R. Hanus, "Kinematic calibration and geometrical parameter identification for robots", IEEE Transactions on robotics and automation, vol. 7, no. 6, December 1991.

[12] ND Digital, http://www.ndigital.com

[13] Advanced Tracking Systems, http://www.atracsys.com

[14] Integrated Surgical Systems, http://www.robodoc.com

[15] Karlsruhe Forschungszentrum, http://presse.fzk.de

[16] Intuitive Surical, http://www.intuitivesurgical.com

[17] Computer Motion, http://www.computermotion.com 